\title{
Thermodynamic products for Sen black hole
}

\author{
Parthapratim Pradhan ${ }^{\mathrm{a}}$ \\ Department of Physics, Vivekananda Satavarshiki Mahavidyalaya (Affiliated to Vidyasagar University), \\ Jhargram, West Midnapur, Manikpara 721513, West Bengal, India
}

Received: 3 June 2015 / Accepted: 23 February 2016 / Published online: 9 March 2016

(C) The Author(s) 2016. This article is published with open access at Springerlink.com

\begin{abstract}
We investigate the properties of inner and outer horizon thermodynamics of Sen black hole (BH) both in Einstein frame $(\mathrm{EF})$ and string frame $(\mathrm{SF})$. We also compute area (or entropy) product, area (or entropy) sum of the said BH in $\mathrm{EF}$ as well as SF. In the EF, we observe that the area (or entropy) product is universal, whereas area (or entropy) sum is not universal. On the other hand, in the SF, area (or entropy) product and area (or entropy) sum don't have any universal behaviour because they all are depends on Arnowitt-DeserMisner (ADM) mass parameter. We also verify that the first law is satisfied at the Cauchy horizon as well as event horizon (EH). In addition, we also compute other thermodynamic products and sums in the EF as well as in the SF. We further compute the Smarr mass formula and Christodoulou's irreducible mass formula for Sen BH. Moreover, we compute the area bound and entropy bound for both the horizons. The upper area bound for EH is actually the Penrose like inequality, which is the first geometric inequality in BHs. Furthermore, we compute the central charges of the left and right moving sectors of the dual CFT in Sen/CFT correspondence using thermodynamic relations. These thermodynamic relations on the multi-horizons give us further understanding the microscopic nature of BH entropy (both interior and exterior).
\end{abstract}

\section{Introduction}

In an un-quantized (classical) general relativity theory any $\mathrm{BH}$ in thermal equilibrium has an entropy and a temperature. Now it is well known by fact that the entropy is proportional to the area of the event horizon (EH) i.e. [1-4]

$\mathcal{S}_{+}=\frac{\mathcal{A}_{+}}{4}$.

where, $\mathcal{S}_{+}$is the Bekenstein-Hawking entropy (in units in which $G=\hbar=c=k=1$ ) and $\mathcal{A}_{+}$is the area of the $\mathrm{EH}$

a e-mails: pppradhan5@ rediffmail.com;pppradhan77@gmail.com
$\left(\mathcal{H}^{+}\right)$. Now this temperature is proportional to the surface gravity of the $\mathcal{H}^{+}$i.e.

$T_{+}=\frac{\kappa_{+}}{2 \pi}$.

where $T_{+}$is the Hawking temperature computed at the $\mathcal{H}^{+}$ and $\kappa_{+}$denotes the surface gravity of the $\mathrm{BH}$ computed at the $\mathcal{H}^{+}$.

In terms of these quantities, the first law of $\mathrm{BH}$ thermodynamics could be expressed as

$\mathrm{d} M=\frac{\kappa_{+}}{8 \pi} \mathrm{d} \mathcal{A}_{+}+\Omega_{+} \mathrm{d} J+\Phi_{+} \mathrm{d} Q$.

It can be seen that $\frac{\kappa_{+}}{8 \pi}$ is analogous to the temperature of $\mathcal{H}^{+}$ in the same way that $\mathcal{A}_{+}$is analogous to entropy. It should be noted that $\frac{\kappa_{+}}{8 \pi}$ and $\mathcal{A}_{+}$are distinct from the temperature and entropy of the BH. and

$\Omega_{+}=\frac{4 \pi J}{M \mathcal{A}_{+}}=\frac{\partial M}{\partial J}$

$\Phi_{+}=\frac{1}{M}\left(\frac{Q}{2}+\frac{2 \pi Q^{3}}{\mathcal{A}_{+}}\right)=\frac{\partial M}{\partial Q}$.

The above relations are computed on the EH only.

It is now well known fact that certain BH has inner horizon or Cauchy horizon $(\mathrm{CH})$ inside the $\mathrm{EH}$ or outer horizon. Naturally, the question should be arises whether similar relations do exist in case of $\mathrm{CH}$ ? It is now well established that the above relations do hold for $\mathrm{CH}\left(\mathcal{H}^{-}\right)$as well as EH. Therefore one may write the inner entropy of the $\mathrm{BH}$ which is proportional to the area of the inner horizon:

$\mathcal{S}_{-}=\frac{\mathcal{A}_{-}}{4}$.

Analogously, the inner Hawking temperature should be calculated via the inner surface gravity of the $\mathrm{BH}$ :

$T_{-}=\frac{\kappa_{-}}{2 \pi}$. 
Using the above inner properties of the $\mathrm{BH}$, we can write the inner first law of $\mathrm{BH}$ thermodynamics

$\mathrm{d} M=-\frac{\kappa_{-}}{8 \pi} \mathrm{d} \mathcal{A}_{-}+\Omega_{-} \mathrm{d} J+\Phi_{-} \mathrm{d} Q$

where,

$\Omega_{-}=\frac{4 \pi J}{M \mathcal{A}_{-}}=\frac{\partial M}{\partial J}$

$\Phi_{-}=\frac{1}{M}\left(\frac{Q}{2}+\frac{2 \pi Q^{3}}{\mathcal{A}_{-}}\right)=\frac{\partial M}{\partial Q}$.

Similarly, the second law is also valid for outer horizon [4] as well as inner horizon which states that

$\mathrm{d} \mathcal{A}_{ \pm} \geq 0$

It has been suggested that every regular axi-symmetric and stationary space-time of Einstein-Maxwell gravity with surrounding matter has a regular $\mathrm{CH}$ inside the $\mathrm{EH}$ if and only if both angular momentum $J$ and charge $Q$ do not vanish. Then the product of the area $A_{ \pm}$of the horizons $\mathcal{H}^{ \pm}$for KerrNewman $(\mathrm{KN})$ class of family could be expressed as by the relation [8]: (see also $[9,10]$ )

$\mathcal{A}_{+} \mathcal{A}_{-}=(8 \pi)^{2}\left(J^{2}+\frac{Q^{4}}{4}\right)$

which is remarkably independent of the ADM mass $(M)$ of the space-time. In the limit $Q=0$, one obtains the area product formula for Kerr BH [12].

Again in string theory and $M$-theory, the product of Killing horizon areas for certain multi-horizon BHs are also independent of the ADM mass. For asymptotically flat BPS (Bogomol'ni-Prasad-Sommerfield) BHs in four and higher dimensions, the quantization rule becomes [13-15]: $\mathcal{A}_{ \pm}=$ $8 \pi \ell_{p l}^{2}\left(\sqrt{N_{1}} \pm \sqrt{N_{2}}\right)$ or

$\mathcal{A}_{+} \mathcal{A}_{-}=\left(8 \pi \ell_{p l}^{2}\right)^{2} N, \quad N \in \mathbb{N}, \quad N_{1} \in \mathbb{N}, \quad N_{2} \in \mathbb{N}$.

where $\ell_{p l}$ is the Planck length, $N_{1}$ and $N_{2}$ are integers for super-symmetric extremal BHs [11,14-18,20-22].

However, it has been well known fact that $\mathrm{CH}$ is an infinite blue-shift region in contrast with $\mathrm{EH}$ (infinite red-shift region). It is also true that $\mathrm{CH}$ is a highly unstable due to the exterior perturbation [23]. Thus there has been indication towards the relevance of $\mathrm{BH} \mathrm{CH}$ in comparison with $\mathrm{EH}$.

Thus in this work, we wish to examine the above mentioned thermodynamical feature of the rotating charged BHs in heterotic string theory [24]. ${ }^{1}$ We have discussed both the situations in Einstein frame (EF) as well as in String frame (SF). The fact that string theory is the leading candidate to unify gravity to other fundamental forces in nature. For this reason, we have chosen the low energy heterotic string theoretical $\mathrm{BH}$. The special characteristics of this string $\mathrm{BH}$ is that they are qualitatively different from those $\mathrm{BH}$ that appear in ordinary Einstein general theory of relativity [5,24]. Most of these solutions are characterized by one or more charges associated with Yang-Mills fields or the anti-symmetric tensor gauge field. Furthermore, this low energy heterotic string $\mathrm{BH}$ carries a finite amount of charge, angular momentum and magnetic dipole moment. It could be produced by twisting method and starting from a rotating $\mathrm{BH}$ having no charge, i.e. the Kerr BH. So, sometimes it is called twisted Kerr BH or Kerr-Sen BH [5].

We prove that in the EF, the area product formula and the entropy product formula are universal, whereas area sum and entropy sum are not universal. Again in the SF, area product, entropy product, area sum and entropy sum formula don't have any universal nature because they all are depends on ADM mass parameter. We also observe that every $\mathrm{BH}$ thermodynamic quantities (e.g. area, entropy, temperature, surface gravity etc.), other than the mass $(M)$, the angular momentum $(J)$ and the charge $(Q)$, can form a quadratic equation whose roots are contained the three basic parameter $M, J, Q$. We further examine that the four laws of $\mathrm{BH}$ mechanics is satisfied at the inner horizon as well as EH. Moreover in EF, we compute the area bound and entropy bound for both the horizons. The upper area bound for $\mathrm{EH}$ is actually the Penrose like inequality, which is the first geometric inequality in BHs [27].

The paper is organized as follows. Section 2 describes the properties of Sen BH in EF and deals with various thermodynamic products. In this section, there are three subsections. In Sect. 2.1, we have discussed the Smarr formula for Sen BH. In Sect. 2.2, we have derived the Christodoulou-Ruffini mass formula for Sen BH. Finally in Sect. 2.3, we have discussed the four laws of BH thermodynamics. In Sect. 3, we computed various thermodynamic products for Sen BH in String frame. Finally, in Sect. 4 we concluded our discussions.

\section{Sen $\mathrm{BH}$ in $\mathbf{E F}$}

An exact rotating charged $\mathrm{BH}$ solution in four dimension heterotic string theory represented by the metric [24] in EF

\footnotetext{
1 The Sen's [24] solutions were later generalized by Sen [25] and also Cvetic and Youm [13]. Since those times one standard class for many investigations of string theory BHs were the four charge solutions parametrized by four boost angles. The Sen 1992 solutions [24] correspond to the special case where three of the four boost angles were taken to vanish. The present manuscript is a special case, where three parameters are taken to vanish.
} 


$$
\begin{aligned}
\mathrm{d} s^{2}= & -\left(1-\frac{2 m r \cosh ^{2} \alpha}{\rho^{2}}\right) \mathrm{d} t^{2} \\
& -\frac{4 a m r \cosh ^{2} \alpha \sin ^{2} \theta}{\rho^{2}} \mathrm{~d} t \mathrm{~d} \phi \\
& +\frac{\rho^{2}}{\Delta} \mathrm{d} r^{2}+\rho^{2} \mathrm{~d} \theta^{2}+\frac{\Upsilon}{\rho^{2}} \sin ^{2} \theta \mathrm{d} \phi^{2}
\end{aligned}
$$

where

$$
\begin{aligned}
\rho^{2} & =r^{2}+a^{2} \cos ^{2} \theta+2 m r \sinh ^{2} \alpha \\
\Delta & =r^{2}-2 m r+a^{2} \\
\Upsilon & =\left(r^{2}+a^{2}+2 m r \sinh ^{2} \alpha\right)^{2}-\Delta a^{2} \sin ^{2} \theta
\end{aligned}
$$

The Maxwell field, dilaton, and anti-symmetric tensor are

$$
\begin{aligned}
& A=\frac{m r \sinh 2 \alpha}{\sqrt{2} \rho^{2}}\left(\mathrm{~d} t-a \sin ^{2} \theta \mathrm{d} \phi\right) \\
& e^{-2 \phi}=\frac{\rho^{2}}{r^{2}+a^{2} \cos ^{2} \theta} \\
& B_{t \phi}=\frac{2 m a r \sinh ^{2} \alpha \sin ^{2} \theta}{\rho^{2}}
\end{aligned}
$$

The above metric describes a $\mathrm{BH}$ solution with mass $M$, charge $Q$, angular momentum $J$, and magnetic dipole moment $\mu$ is given by

$$
\begin{aligned}
M & =\frac{m}{2}(1+\cosh 2 \alpha) \\
Q & =\frac{m}{\sqrt{2}} \sinh 2 \alpha \\
J & =\frac{m a}{2}(1+\cosh 2 \alpha) \\
\mu & =\frac{1}{\sqrt{2}} m a \sinh 2 \alpha
\end{aligned}
$$

Since we shall analyze various thermodynamic products of this $\mathrm{BH}$, for this purpose it will be more convenient to write $m, a$ and $\alpha$ in terms of the independent physical parameters $M, J$ and $Q$ inverting the relations given in Eq. (24). Thus we find

$m=M-\frac{Q^{2}}{2 M}$

$\sinh 2 \alpha=\frac{2 \sqrt{2} Q M}{\left(2 M^{2}-Q^{2}\right)}$

$a=\frac{J}{M}$

This is the well known Sen BH solution [24] which was discovered by Sen in 1992.

There are two horizons for Sen $\mathrm{BH}$ namely $\mathrm{EH}\left(\mathcal{H}^{+}\right)$or outer horizon and $\mathrm{CH}\left(\mathcal{H}^{-}\right)$or inner horizon. Their radius can be determined by solving the following metric function as

$$
\Delta \equiv \Delta(r)=r^{2}-\left(2 M-\frac{Q^{2}}{M}\right) r+a^{2}=0 .
$$

which gives

$r_{ \pm}=\left(M-\frac{Q^{2}}{2 M}\right) \pm \sqrt{\left(M-\frac{Q^{2}}{2 M}\right)^{2}-a^{2}}$

Here $r_{+}$is called $\mathrm{EH}$ and $r_{-}$is called $\mathrm{CH}$. It may be noted that $r_{+}>r_{-}$. Interestingly, the solution of the Eq. (28) gives

$r_{+}+r_{-}=2 M-\frac{Q^{2}}{M}$ and $r_{+} r_{-}=a^{2}$.

This indicates that the sum and product of the horizon radii depends on the mass parameter.

From Eq. (29) one can see that the horizon disappears unless

$a \leq\left(M-\frac{Q^{2}}{2 M}\right)$

Thus the extremal limit of the Sen BH corresponds to

$a=\left(M-\frac{Q^{2}}{2 M}\right)$.

and the horizon for extremal Sen $\mathrm{BH}$ is situated at

$r_{\mathrm{ex}}=r_{+}=r_{-}=a=\left(M-\frac{Q^{2}}{2 M}\right)$.

Now we would like to compute various thermodynamic quantities of the Sen BH. The area [2,3] of both the horizon $\left(\mathcal{H}^{ \pm}\right)$in EF is

$$
\begin{aligned}
\mathcal{A}_{ \pm} & =\int_{0}^{2 \pi} \int_{0}^{\pi} \sqrt{g_{\theta \theta} g_{\phi \phi}} \mathrm{d} \theta \mathrm{d} \phi \\
& =8 \pi M\left[\left(M-\frac{Q^{2}}{2 M}\right) \pm \sqrt{\left(M-\frac{Q^{2}}{2 M}\right)^{2}-a^{2}}\right]
\end{aligned}
$$

The angular velocity of $\mathcal{H}^{ \pm}$computed at the horizon is given by

$$
\Omega_{ \pm}=\frac{J}{2 M^{2}\left[\left(M-\frac{Q^{2}}{2 M}\right) \pm \sqrt{\left(M-\frac{Q^{2}}{2 M}\right)^{2}-a^{2}}\right]}
$$

The semi-classical Bekenstein-Hawking entropy of $\mathcal{H}^{ \pm}$ reads

$$
\mathcal{S}_{ \pm}=2 \pi M\left[\left(M-\frac{Q^{2}}{2 M}\right) \pm \sqrt{\left(M-\frac{Q^{2}}{2 M}\right)^{2}-a^{2}}\right]
$$


The surface gravity [24] of $\mathcal{H}^{ \pm}$is given by

$$
\kappa_{ \pm}= \pm \frac{\sqrt{\left(2 M^{2}-Q^{2}\right)^{2}-4 J^{2}}}{2 M\left[\left(2 M^{2}-Q^{2}\right) \pm \sqrt{\left(2 M^{2}-Q^{2}\right)^{2}-4 J^{2}}\right]} .
$$

and

$\kappa_{+}>\kappa_{-}$

and the $\mathrm{BH}$ temperature or Hawking temperature of $\mathcal{H}^{ \pm}$reads as

$$
T_{ \pm}= \pm \frac{\sqrt{\left(2 M^{2}-Q^{2}\right)^{2}-4 J^{2}}}{4 \pi M\left[\left(2 M^{2}-Q^{2}\right) \pm \sqrt{\left(2 M^{2}-Q^{2}\right)^{2}-4 J^{2}}\right]} .
$$

It should be noted that $T_{+}>T_{-}$.

The Komar [26] energy for $\mathcal{H}^{ \pm}$is given by

$E_{ \pm}= \pm \sqrt{\left(2 M^{2}-Q^{2}\right)^{2}-4 J^{2}}$

Finally, the horizon Killing vector field may be defined for $\mathcal{H}^{ \pm}$as

$\chi_{ \pm}{ }^{a}=\left(\partial_{t}\right)^{a}+\Omega_{ \pm}\left(\partial_{\phi}\right)^{a}$.

Now we shall see that every BH thermodynamic quantities (e.g. area, entropy, temperature, surface gravity etc.), other than the mass $(M)$, the angular momentum $(J)$ and the charge $(Q)$, which is also defined on $\mathcal{H}^{ \pm}$can form a quadratic equation of thermodynamic quantities like horizon radii $\left(r_{ \pm}\right)$.

Firstly, we compute the "product" and "sum" of the inner horizon area and outer horizon area as

$\mathcal{A}_{-} \mathcal{A}_{+}=(8 \pi J)^{2}$.

and

$\mathcal{A}_{-}+\mathcal{A}_{+}=8 \pi\left(2 M^{2}-Q^{2}\right)$.

Interestingly, the area sum and the area product might be satisfied the following quadratic equation:

$\mathcal{A}^{2}-8 \pi\left(2 M^{2}-Q^{2}\right) \mathcal{A}+(8 \pi J)^{2}=0$.

With the help of the above Eqs. (43) and (44), we can easily see that the "area product" is universal, while the "area sum" is not universal for Sen BH in EF because it depends on the $\mathrm{BH}$ mass or ADM mass parameter. For completeness, we further compute the area minus and area division, which is given by

$\mathcal{A}_{ \pm}-\mathcal{A}_{\mp}=8 \pi M T_{ \pm} \mathcal{A}_{ \pm}$.

and

$\frac{\mathcal{A}_{+}}{\mathcal{A}_{-}}=\frac{r_{+}}{r_{-}}=\frac{\Omega_{-}}{\Omega_{+}}=-\frac{T_{-}}{T_{+}}$.
Again, the sum of area inverse is found to be

$\frac{1}{\mathcal{A}_{+}}+\frac{1}{\mathcal{A}_{-}}=\frac{2 M^{2}-Q^{2}}{8 \pi J^{2}}$.

and the minus of area inverse is computed to be

$\frac{1}{\mathcal{A}_{ \pm}}-\frac{1}{\mathcal{A}_{\mp}}=\mp \frac{\sqrt{\left(2 M^{2}-Q^{2}\right)^{2}-4 J^{2}}}{8 \pi J^{2}}$.

It indicates that they all are mass dependent relations.

Likewise, the "entropy product" and "entropy sum" of $\mathcal{H}^{ \pm}$ becomes:

$\mathcal{S}_{-} \mathcal{S}_{+}=(2 \pi J)^{2}$.

and

$\mathcal{S}_{-}+\mathcal{S}_{+}=2 \pi\left(2 M^{2}-Q^{2}\right)$.

The quadratic equation of entropy becomes

$\mathcal{S}^{2}-2 \pi\left(2 M^{2}-Q^{2}\right) \mathcal{S}+(2 \pi J)^{2}=0$.

It indicates that "entropy product" is independent of mass and "entropy sum" depends on the BH mass.

Using Eq. (47), one can derive another important relations:

$T_{+} \mathcal{S}_{+}+T_{-} \mathcal{S}_{-}=0$.

and

$\frac{\Omega_{+}}{T_{+}}+\frac{\Omega_{-}}{T_{-}}=0$.

The above important thermodynamic products of multi horizons may be used to determine the classical BH entropy in terms of Cardy formula, therefore giving some evidence for a $\mathrm{BH} / \mathrm{CFT}$ description of the corresponding microstates [18]. It has been also shown that from the above Eq. (53), the central charge being the same for two horizon BHs. Explicit calculation of the central charges $c_{L}=c_{R}=12 \mathrm{~J}$ using Cardy formula has been done in Appendix B. Using thermodynamical relations, we derive the dimensionless temperature of microscopic CFT, which is perfect agreement with the ones derived from hidden conformal symmetry in the low frequency scattering off the $\mathrm{BH}$ [19].

Based on these above relations, we would like to compute the entropy bound of $\mathcal{H}^{ \pm}$which is exactly Penrose-like inequality for event horizon. From the Eq. (31), we obtain Kerr like bound for Sen BH:

$M^{4}-Q^{2} M^{2}+\frac{Q^{4}-4 J^{2}}{4} \geq 0$.

or

$M^{2} \geq J+\frac{Q^{2}}{2}$.

Since $r_{+} \geq r_{-}$thus $\mathcal{S}_{+} \geq \mathcal{S}_{-} \geq 0$. Then the entropy product (50) gives: 
$\mathcal{S}_{+} \geq \sqrt{\mathcal{S}_{+} \mathcal{S}_{-}}=2 \pi J \geq \mathcal{S}_{-}$.

and the entropy sum gives:

$$
\begin{aligned}
2 \pi\left(2 M^{2}-Q^{2}\right) & =\mathcal{S}_{+}+\mathcal{S}_{-} \geq \mathcal{S}_{+} \geq \frac{\mathcal{S}_{+}+\mathcal{S}_{-}}{2} \\
& =\pi\left(2 M^{2}-Q^{2}\right) \geq \mathcal{S}_{-} .
\end{aligned}
$$

Thus the entropy bound for $\mathcal{H}^{+}$:

$\pi\left(2 M^{2}-Q^{2}\right) \leq \mathcal{S}_{+} \leq 2 \pi\left(2 M^{2}-Q^{2}\right)$.

and the entropy bound for $\mathcal{H}^{-}$:

$0 \leq \mathcal{S}_{-} \leq 2 \pi J$.

From this bound, we can derive area bound which could be found in the latter section. It should be noted that in the limit $Q=0$, we obtain the Kerr entropy bound [28].

Similarly, we can obtain the "product of surface gravity" and "sum of surface gravity" of $\mathcal{H}^{ \pm}$is

$\kappa_{-} \kappa_{+}=-\frac{\left(2 M^{2}-Q^{2}\right)^{2}-4 J^{2}}{(4 J M)^{2}}$.

and

$\kappa_{-}+\kappa_{+}=-\frac{\left(2 M^{2}-Q^{2}\right)^{2}-4 J^{2}}{4 M J^{2}}$.

It suggests that surface gravity product and surface gravity sum are not universal. It may be noted that surface gravity satisfied the following quadratic equation.

$$
\begin{aligned}
& \kappa^{2}-\left(\frac{4 J^{2}-\left(2 M^{2}-Q^{2}\right)^{2}}{4 M J^{2}}\right) \kappa \\
& +\left(\frac{4 J^{2}-\left(2 M^{2}-Q^{2}\right)^{2}}{4 M J^{2}}\right)=0 .
\end{aligned}
$$

Similarly, one can obtain "surface temperature product" and "surface temperature sum" of $\mathcal{H}^{ \pm}$as follows

$T_{-} T_{+}=-\frac{\left(2 M^{2}-Q^{2}\right)^{2}-4 J^{2}}{(8 \pi J M)^{2}}$.

and

$T_{-}+T_{+}=-\frac{\left(2 M^{2}-Q^{2}\right)^{2}-4 J^{2}}{8 \pi M J^{2}}$.

It seems that these products and sum are not universal.

Finally, "Komar energy product" and "Komar energy sum" of $\mathcal{H}^{ \pm}$for Sen BH is given by

$$
\begin{aligned}
E_{+} E_{-} & =\left(2 \mathcal{S}_{+} T_{+}\right)\left(2 \mathcal{S}_{-} T_{-}\right) \\
& =-\left[\left(2 M^{2}-Q^{2}\right)^{2}-4 J^{2}\right] .
\end{aligned}
$$

and

$$
E_{+}+E_{-}=\left(2 \mathcal{S}_{+} T_{+}\right)+\left(2 \mathcal{S}_{-} T_{-}\right)=0 .
$$

The above calculation suggests that the product of the area and entropy of $\mathcal{H}^{ \pm}$are proportional to the square of the spin parameter $J$. Surface gravity product, surface temperature product and Komar energy product depends on ADM mass. Thus, we may conclude that they are not universal except the area product and entropy product. In appendix $\mathrm{A}$, we have computed various thermodynamic parameters for $\mathrm{KN} \mathrm{BH}$, Kerr BH in comparison with Sen BH. Now we are going to derive the Smarr formula for Sen $\mathrm{BH}$.

\subsection{Smarr formula for Sen BH}

It is well known that for $\mathrm{KN} \mathrm{BH}$ the area of the outer [7] and inner horizons are

$\mathcal{A}_{ \pm}=4 \pi\left(2 M^{2}-Q^{2} \pm 2 \sqrt{M^{4}-J^{2}-M^{2} Q^{2}}\right)$.

Indeed, it is constant over the $\mathcal{H}^{ \pm}$. Similarly, we can evaluate the area of $\mathcal{H}^{ \pm}$for Sen BH reads

$\mathcal{A}_{ \pm}=8 \pi M\left[\left(M-\frac{Q^{2}}{2 M}\right) \pm \sqrt{\left(M-\frac{Q^{2}}{2 M}\right)^{2}-a^{2}}\right]$.

Inverting the above relation one can compute the $\mathrm{BH}$ mass or ADM mass can be expressed in terms of area of both the horizon.

$M^{2}=\frac{\mathcal{A}_{ \pm}}{16 \pi}+\frac{4 \pi J^{2}}{\mathcal{A}_{ \pm}}+\frac{Q^{2}}{2}$.

It is remarkable that the mass can be expressed as in terms of both area of $\mathcal{H}^{+}$and $\mathcal{H}^{-}$. Now we will see what happens with the mass differential? It could be also expressed as three physical invariants of both $\mathcal{H}^{+}$and $\mathcal{H}^{-}$,

$\mathrm{d} M=\Gamma_{ \pm} \mathrm{d} \mathcal{A}_{ \pm}+\Omega_{ \pm} \mathrm{d} J+\Phi_{ \pm} \mathrm{d} Q$.

where

$$
\begin{aligned}
& \Gamma_{ \pm}=\frac{\partial M}{\partial \mathcal{A}_{ \pm}}=\frac{1}{M}\left(\frac{1}{32 \pi}-\frac{2 \pi J^{2}}{\mathcal{A}_{ \pm}^{2}}\right) \\
& \Omega_{ \pm}=\frac{\partial M}{\partial J}=\frac{4 \pi J}{M \mathcal{A}_{ \pm}}=\frac{a}{2 M r_{ \pm}} \\
& \Phi_{ \pm}=\frac{\partial M}{\partial Q}=\frac{Q}{M} .
\end{aligned}
$$

where

$\Gamma_{ \pm}=$Effective surface tension for $\mathcal{H}^{+}$and $\mathcal{H}^{-}$

$\Omega_{ \pm}=$Angular velocity for $\mathcal{H}^{ \pm}$

$\Phi_{ \pm}=$Electromagnetic potentials for $\mathcal{H}^{ \pm}$ 
The effective surface tension can be rewritten as

$$
\begin{aligned}
\Gamma_{ \pm} & =\frac{1}{M}\left(\frac{1}{32 \pi}-\frac{2 \pi J^{2}}{\mathcal{A}_{ \pm}^{2}}\right) \\
& =\frac{1}{32 \pi M}\left(1-\frac{64 \pi^{2} J^{2}}{\mathcal{A}_{ \pm}^{2}}\right) \\
& =\frac{1}{32 \pi M}\left(1-\frac{a^{2}}{r_{ \pm}^{2}}\right) \\
& =\frac{r_{ \pm}-M}{32 \pi M r_{ \pm}}=\frac{\kappa_{ \pm}}{8 \pi}
\end{aligned}
$$

where $\kappa_{ \pm}$is the surface gravity of $\mathcal{H}^{ \pm}$as previously defined.

Thus the mass can be expressed in terms of these quantities both for $\mathcal{H}^{ \pm}$as a simple bilinear form

$M=2 \Gamma_{ \pm} \mathcal{A}_{ \pm}+2 J \Omega_{ \pm}+2 Q \Phi_{ \pm}$

This has been derived from the homogenous function of degree $\frac{1}{2}$ in $\left(\mathcal{A}_{ \pm}, J, Q\right)$. Remarkably, $\Gamma_{ \pm}, \Omega_{ \pm}$and $\Phi_{ \pm}$are constant on the $\mathcal{H}^{+}$and $\mathcal{H}^{-}$for any stationary, axially symmetric space-time.

Since the $\mathrm{d} M$ is a total perfect differential, one may choose freely any path of integration in $\left(\mathcal{A}_{ \pm}, J, Q\right)$ space. Thus one could define surface energy $\mathcal{E}_{s, \pm}$ for $\mathcal{H}^{ \pm}$

$\mathcal{E}_{s, \pm}=\int_{0}^{\mathcal{A}_{ \pm}} \Gamma\left(\tilde{\mathcal{A}_{ \pm}}, 0,0\right) \mathrm{d} \tilde{\mathcal{A}_{ \pm}}$

the rotational energy for $\mathcal{H}^{ \pm}$can be defined by

$\mathcal{E}_{r, \pm}=\int_{0}^{J} \Omega_{ \pm}\left(\mathcal{A}_{ \pm}, \tilde{J}, 0\right) \mathrm{d} \tilde{J}, \mathcal{A}_{ \pm}$fixed;

and the electromagnetic energy for $\mathcal{H}^{ \pm}$is

$\mathcal{E}_{e m, \pm}=\int_{0}^{Q} \Phi\left(\mathcal{A}_{ \pm}, J, \tilde{Q}\right) \mathrm{d} \tilde{Q}, \mathcal{A}_{ \pm}, J$ fixed

Therefore, we may rewrite the Eq. (75) as

$M= \pm \frac{\kappa_{ \pm}}{4 \pi} \mathcal{A}_{ \pm}+2 J \Omega_{ \pm}+2 Q \Phi_{ \pm}$.

or

$M-2 J \Omega_{ \pm}-2 Q \Phi_{ \pm}= \pm \frac{\kappa_{ \pm}}{4 \pi} \mathcal{A}_{ \pm}$.

or

$M-2 J \Omega_{ \pm}-2 \Phi_{ \pm} Q= \pm \frac{T_{ \pm}}{2} \mathcal{A}_{ \pm}$.

or

$\frac{M}{2}= \pm T_{ \pm} \mathcal{S}_{ \pm}+J \Omega_{ \pm}+Q \Phi_{ \pm}$.

This could be recognized as a generalized Smarr-GibbsDuhem relation on $\mathcal{H}^{ \pm}$for Sen $\mathrm{BH}$.

\subsection{Irreducible mass product for Sen $\mathrm{BH}$}

In this section, we will derive Christodoulou and Ruffini [29] mass formula for Sen BH. Christodoulou had shown that the irreducible mass $M_{\text {irr }}$ of a Kerr BH is related to the surface area $\mathcal{A}$ of the $\mathrm{BH}$ by the following formula

$M_{\mathrm{irr}}^{2}=\frac{\mathcal{A}}{16 \pi}$.

It is now well known that this formula is valid for both the horizons. Thus we can define it for $\mathcal{H}^{ \pm}$:

$M_{\mathrm{irr}, \pm}^{2}=\frac{\mathcal{A}_{ \pm}}{16 \pi}=\frac{M r_{ \pm}}{2}$

where ' + ' sign indicates for $\mathcal{H}^{+}$and '- ' indicates for $\mathcal{H}^{-}$.

Likewise, the area and angular velocity may be expressed in terms of $M_{\text {irr, }}$ :

$\mathcal{A}_{ \pm}=16 \pi\left(M_{\mathrm{irr}, \pm}\right)^{2}$.

and

$\Omega_{ \pm}=\frac{a}{4\left(M_{\text {irr }, \pm}\right)^{2}}$.

Interestingly, the product of the irreducible mass of $\mathcal{H}^{ \pm}$for Sen $\mathrm{BH}$ is universal.

$M_{\text {irr, }+} M_{\text {irr,- }}=\frac{J}{2}$.

The Christodoulou-Ruffini mass formula for Sen BH and for both the horizon $\left(\mathcal{H}^{ \pm}\right)$reads as:

$M^{2}=\left(M_{\mathrm{irr}, \pm}+\frac{Q^{2}}{4 M_{\mathrm{irr}, \pm}}\right)+\frac{J^{2}}{4\left(M_{\mathrm{irr}, \pm}\right)^{2}}$.

Based on the above relations, we would like to compute the area bound and irreducible mass bound for Sen $\mathrm{BH}$ followed by the previous section. Since $r_{+} \geq r_{-}$, one obtains $\mathcal{A}_{+} \geq$ $\mathcal{A}_{-} \geq 0$. Therefore the area product gives:

$\mathcal{A}_{+} \geq \sqrt{\mathcal{A}_{+} \mathcal{A}_{-}}=8 \pi J \geq \mathcal{A}_{-}$.

and the area sum gives:

$$
\begin{aligned}
8 \pi\left(2 M^{2}-Q^{2}\right) & =\mathcal{A}_{+}+\mathcal{A}_{-} \geq \mathcal{A}_{+} \geq \frac{\mathcal{A}_{+}+\mathcal{A}_{-}}{2} \\
& =4 \pi\left(2 M^{2}-Q^{2}\right) \geq \mathcal{A}_{-} .
\end{aligned}
$$

Thus the area bound for $\mathcal{H}^{+}$:

$4 \pi\left(2 M^{2}-Q^{2}\right) \leq \mathcal{A}_{+} \leq 8 \pi\left(2 M^{2}-Q^{2}\right)$.

and the area bound for $\mathcal{H}^{-}$:

$0 \leq \mathcal{A}_{-} \leq 8 \pi J$

From this area bound, we get irreducible mass bound for Sen $\mathrm{BH}$ : for $\mathcal{H}^{+}$: 
$\frac{\sqrt{2 M^{2}-Q^{2}}}{2} \leq M_{\mathrm{irr},+} \leq \frac{\sqrt{2 M^{2}-Q^{2}}}{\sqrt{2}}$.

and for $\mathcal{H}^{-}$:

$0 \leq M_{\text {irr, }-} \leq \sqrt{\frac{J}{2}}$.

Equation (93) is nothing but the Penrose inequality, which is the first geometric inequality for BHs [27].

\subsection{The four Laws of BH thermodynamics on $\mathcal{H}^{ \pm}$}

Let us quickly examine the four laws of $\mathrm{BH}$ thermodynamics for Sen BH. For KN BH, Bardeen et al. [4] formulated the black hole thermodynamics for the $\mathrm{EH}$ which is analogous to the classical laws of thermodynamics. We derive here same for Sen BH both on the $\mathrm{EH}$ as well as $\mathrm{CH}$. We have already been derived the surface gravity in the previous section given by the Eqs. (38) and (40). Using this two equations we can easily say that the surface gravity and the surface temperature are constant on the $\mathcal{H}^{ \pm}$and therefore, it is remarkable that the Zeroth law of $\mathrm{BH}$ thermodynamics holds for $\mathrm{CH}$ as well as $\mathrm{EH}$.

- The Zeroth Law: The surface gravity, $\kappa_{ \pm}$of a stationary black hole is constant over the $\mathrm{EH}$ as well as $\mathrm{CH}$.

Quite similarly, the first law of BH thermodynamics is also satisfied not only at the outer horizon but also at the inner horizon.

- The first law: Any perturbation of a stationary BHs, the change of mass (change of energy) is related to change of mass, angular momentum, and electric charge by:

$$
\mathrm{d} M= \pm \frac{\kappa_{ \pm}}{8 \pi} \mathrm{d} \mathcal{A}_{ \pm}+\Omega_{ \pm} \mathrm{d} J+\Phi_{ \pm} \mathrm{d} Q
$$

It can be seen that $\frac{\kappa_{ \pm}}{8 \pi}$ is analogous to the temperature of $\mathcal{H}^{ \pm}$in the same way that $\mathcal{A}_{ \pm}$is analogous to entropy. It should be noted that $\frac{\kappa_{ \pm}}{8 \pi}$ and $\mathcal{A}_{ \pm}$are quite distinct from the temperature and entropy of the $\mathrm{BH}$.

Again, the second law of BH thermodynamics is also satisfied both on the inner horizon and outer horizon.

- The second law: The area $\mathcal{A}_{ \pm}$of both EH and $\mathrm{CH}$ never decreases, i.e.

$$
\mathrm{d} \mathcal{A}_{ \pm}=\frac{4 \mathcal{A}_{ \pm}}{r_{ \pm}-r_{\mp}}\left(\mathrm{d} M-\boldsymbol{\Omega}_{ \pm} . \mathrm{d} \mathbf{J}-\Phi_{ \pm} \mathrm{d} Q\right) \geq 0
$$

or

$$
\mathrm{d} M_{\mathrm{irr}, \pm}=\frac{2 M_{\mathrm{irr}, \pm}}{r_{ \pm}-r_{\mp}}\left(\mathrm{d} M-\boldsymbol{\Omega}_{ \pm} . \mathrm{d} \mathbf{J}-\Phi_{ \pm} \mathrm{d} Q\right) \geq 0
$$

The change in irreducible mass of both $\mathrm{EH}$ and $\mathrm{CH}$ can never be negative. It follows immediately that

$$
\mathrm{d} M>\boldsymbol{\Omega}_{ \pm} \cdot \mathrm{d} \mathbf{J}+\Phi_{ \pm} \mathrm{d} Q
$$

For the extremal Sen $\mathrm{BH}\left(r_{+}=r_{-}\right)$, we have $T_{+}=T_{-}=$ $0=\kappa_{+}=\kappa_{-}$. Therefore the third law becomes:

- The third law: It is impossible by any mechanism, no matter how idealized, to reduce, $\kappa_{ \pm}$the surface gravity of both $\mathrm{EH}$ and $\mathrm{CH}$ to zero by a finite number of operations.

Thus we have checked that the four laws of $\mathrm{BH}$ mechanics satisfied on $\mathrm{CH}$ as well as $\mathrm{EH}$.

So far all the computations have been carried out for Sen $\mathrm{BH}$ in EF. Now we will see in next section, what happens for these computations for Sen BH in SF?

\section{Sen $\mathrm{BH}$ in $\mathrm{SF}$}

This frame sometimes used because in this frame the physical degrees of freedom move along the geodesics of the metric [6]. Therefore the corresponding metric in the SF and the EF are conformally related by the following relation

$G_{a b}=e^{2 \phi} g_{a b}$.

where $G_{a b}$ are the covariant components of the metric in the SF, $g_{a b}$ are the components of the metric in the EF and $\phi$ is the dilation field. For contravariant components they are related by

$$
G^{a b}=e^{-2 \phi} g^{a b} .
$$

The dilation field is given by

$e^{2 \phi}=\frac{r^{2}+a^{2} \cos ^{2} \theta}{\rho^{2}}$.

For simplicity, we denote

$\chi=r^{2}+a^{2} \cos ^{2} \theta$.

First, we need to write the metric for Sen BH in SF [5], which is given by

$$
\begin{aligned}
\mathrm{d} s^{2}= & -\frac{\chi}{\rho^{2}}\left(1-\frac{2 m r \cosh ^{2} \alpha}{\rho^{2}}\right) \mathrm{d} t^{2} \\
& -\frac{4 a m r \chi \cosh ^{2} \alpha \sin ^{2} \theta}{\rho^{4}} \mathrm{~d} t \mathrm{~d} \phi+\frac{\chi}{\Delta} \mathrm{d} r^{2}+\chi \mathrm{d} \theta^{2} \\
& +\frac{\chi}{\rho^{4}} \Upsilon \sin ^{2} \theta \mathrm{d} \phi^{2}
\end{aligned}
$$


Since the action in SF is different from EF therefore the conserved quantities are also different. Now we define this conserved quantities (mass, charge, angular momentum) in $\mathrm{SF}$ are $\mathcal{M}, \mathcal{Q}$ and $\mathcal{J}$ respectively. Then the horizon radii in SF becomes

$r_{ \pm}^{\mathrm{SF}}=\left(\mathcal{M}-\frac{\mathcal{Q}^{2}}{2 \mathcal{M}}\right) \pm \sqrt{\left(\mathcal{M}-\frac{\mathcal{Q}^{2}}{2 \mathcal{M}}\right)^{2}-a^{2}}$.

Here the spin parameter $a=\frac{\mathcal{J}}{\mathcal{M}}$.

Now the area of both the horizons $\left(\mathcal{H}^{ \pm}\right)$in SF is given by

$$
\begin{aligned}
\mathcal{A}_{ \pm}^{\mathrm{SF}}= & 4 \pi\left[r_{ \pm}^{\mathrm{SF}}\left(r_{ \pm}^{\mathrm{SF}}+b\right)+a^{2}\right] \\
\times & {\left[1-\frac{b r_{ \pm}^{\mathrm{SF}}}{a \sqrt{r_{ \pm}^{\mathrm{SF}}\left(r_{ \pm}^{\mathrm{SF}}+b\right)}}\right.} \\
& \left.\times \tan ^{-1} \frac{a}{\sqrt{r_{ \pm}^{\mathrm{SF}}\left(r_{ \pm}^{\mathrm{SF}}+b\right)}}\right] .
\end{aligned}
$$

where, $b=\frac{\mathcal{Q}^{2}}{\mathcal{M}}, G_{\theta \theta}=\chi$ and $G_{\phi \phi}=\frac{\chi}{\rho^{4}} \Upsilon \sin ^{2} \theta$. Similarly, we can compute the entropy for both the horizons $\left(\mathcal{H}^{ \pm}\right)$in the SF:

$$
\begin{aligned}
\mathcal{S}_{ \pm} \mathrm{SF}= & \pi\left[r_{ \pm}^{\mathrm{SF}}\left(r_{ \pm}^{\mathrm{SF}}+b\right)+a^{2}\right] \\
\times & {\left[1-\frac{r_{ \pm}^{\mathrm{SF}} b}{a \sqrt{r_{ \pm}^{\mathrm{SF}}\left(r_{ \pm}^{\mathrm{SF}}+b\right)}}\right.} \\
& \left.\times \tan ^{-1} \frac{a}{\sqrt{r_{ \pm}^{\mathrm{SF}}\left(r_{ \pm}^{\mathrm{SF}}+b\right)}}\right] .
\end{aligned}
$$

Now we turn to the most interesting case that is the "Area product" for Sen BH in SF:

$$
\begin{aligned}
& \mathcal{A}_{+}{ }^{\mathrm{SF}} \mathcal{A}_{-}{ }^{\mathrm{SF}}=(8 \pi \mathcal{J})^{2} \\
& \times\left[1-\frac{b r_{+}^{\mathrm{SF}}}{a \sqrt{r_{+}^{\mathrm{SF}}\left(r_{+}^{\mathrm{SF}}+b\right)}} \tan ^{-1} \frac{a}{\sqrt{r_{+}^{\mathrm{SF}}\left(r_{+}^{\mathrm{SF}}+b\right)}}\right] \\
& \times\left[1-\frac{b r_{-}^{\mathrm{SF}}}{a \sqrt{r_{-}^{\mathrm{SF}}\left(r_{-}^{\mathrm{SF}}+b\right)}} \tan ^{-1} \frac{a}{\sqrt{r_{-}^{\mathrm{SF}}\left(r_{-}^{\mathrm{SF}}+b\right)}}\right] .
\end{aligned}
$$

Interestingly, it seems that the product of horizon area of $\mathcal{H}^{ \pm}$in SF for Sen BH is not universal. This is one of the key result of the work. The result in the SF is quite different from the EF due to the fact that the action in SF is quite different from the EF, therefore the corresponding conserved quantities should be different. Actually, when the $M, J$ and $Q$ are computed in EF the action should be Einstein-Hilbert type andwhere the ADM formulas have been used, whereas when the action is written in SF, the corresponding quantities are very likely to be different. Therefore the parameters $M$, $J$ in particular could no longer be identified with the conserved charges associated with the time-translation and the rotational symmetry.

But if we expand the function of $\tan ^{-1} x$ as

$\tan ^{-1} x=x-\frac{x^{3}}{3}+\frac{x^{5}}{5}-\frac{x^{7}}{7}+\cdots$.

then we find the the area of both the horizons $\left(\mathcal{H}^{ \pm}\right)$as

$$
\begin{aligned}
\mathcal{A}_{ \pm} \mathrm{SF}= & \frac{8 \pi \mathcal{M}\left(r_{ \pm}^{\mathrm{SF}}\right)^{2}}{\left(r_{ \pm}^{\mathrm{SF}}+b\right)}\left[1+\frac{b}{3\left(r_{ \pm}^{\mathrm{SF}}+b\right)}\left(\frac{a}{r_{ \pm}^{\mathrm{SF}}}\right)^{2}\right. \\
& \left.-\frac{b}{5\left(r_{ \pm}^{\mathrm{SF}}+b\right)^{2}}\left(\frac{a}{r_{ \pm}^{\mathrm{SF}}}\right)^{4}+\mathcal{O}\left(\frac{a}{r_{ \pm}^{\mathrm{SF}}}\right)^{6}\right] .
\end{aligned}
$$

It follows from the above equation it is very difficult to find the exact mass parameter in terms of the area of $\mathcal{H}^{ \pm}$in SF. Therefore due to same reasons it is also quite difficult to find the Hawking temperature from the mass differential. So one way we could find the Hawking temperature in SF by using the formula as used Sen in [24]:

$T^{\mathrm{SF}}=\frac{\kappa^{\mathrm{SF}}}{2 \pi}=\left.\frac{\lim _{r \rightarrow r_{ \pm}^{\mathrm{SF}}} \sqrt{G^{r r}} \partial_{r} \sqrt{-G_{t t}}}{2 \pi}\right|_{\theta=0}$

which gives on the $\mathcal{H}^{ \pm}$

$T_{ \pm}^{\mathrm{SF}}=\frac{2\left(r_{ \pm}^{\mathrm{SF}}-\mathcal{M}\right)+b}{4 \pi\left[r_{ \pm}^{\mathrm{SF}}\left(r_{ \pm}^{\mathrm{SF}}+b\right)+a^{2}\right]}$.

Similarly, we could find the angular velocity by using the formula

$\Omega^{\mathrm{SF}}=\frac{-G_{t \phi}+\sqrt{G_{t \phi}^{2}-G_{\phi \phi} G_{t t}}}{G_{\phi \phi}}$.

On the horizon the angular velocity could be written as

$\Omega_{ \pm}^{\mathrm{SF}}=-\frac{G_{t \phi}}{G_{\phi \phi}}=\frac{2 a \mathcal{M} r_{ \pm}^{\mathrm{SF}}}{\left[r_{ \pm}^{\mathrm{SF}}\left(r_{ \pm}^{\mathrm{SF}}+b\right)+a^{2}\right]^{2}}$.

Now we can write the first law of thermodynamics in the SF as

$\mathrm{d} \mathcal{M}= \pm T_{ \pm}^{\mathrm{SF}} \mathrm{d} \mathcal{S}_{ \pm}^{\mathrm{SF}}+\Omega_{ \pm}^{\mathrm{SF}} \mathrm{d} \mathcal{J}+\cdots$

Now it implies that the $\mathrm{BH}$ temperature, angular velocity and probably electric potentials (since charge is different) in SF are quite different from EF because the action and metric are different as we have discussed previously. This is why the area (or entropy) product relation in two frames are quite distinct. 
We also note that the sum of horizon area in SF reads

$$
\begin{aligned}
& \mathcal{A}_{+}{ }^{\mathrm{SF}}+\mathcal{A}_{-}^{\mathrm{SF}}=8 \pi \mathcal{M} r_{+}{ }^{\mathrm{SF}} \\
& \times\left[1-\frac{b r_{+}^{\mathrm{SF}}}{a \sqrt{r_{+}^{\mathrm{SF}}\left(r_{+}^{\mathrm{SF}}+b\right)}} \tan ^{-1} \frac{a}{\sqrt{r_{+}^{\mathrm{SF}}\left(r_{+}^{\mathrm{SF}}+b\right)}}\right] \\
& +8 \pi \mathcal{M} r_{-} \mathrm{SF}\left[1-\frac{b r_{-}^{\mathrm{SF}}}{a \sqrt{r_{-}^{\mathrm{SF}}\left(r_{-}^{\mathrm{SF}}+b\right)}} \tan ^{-1} \frac{a}{\sqrt{r_{-}^{\mathrm{SF}}\left(r_{-}^{\mathrm{SF}}+b\right)}}\right] .
\end{aligned}
$$

Like-wise, the entropy product and entropy sum for Sen $\mathrm{BH}$ in $\mathrm{SF}$ is

$$
\begin{aligned}
\mathcal{S}_{+}{ }^{\mathrm{SF}} \mathcal{S}_{-}{ }^{\mathrm{SF}}=(2 \pi \mathcal{J})^{2} \\
\times\left[1-\frac{b r_{+}^{\mathrm{SF}}}{a \sqrt{r_{+}^{\mathrm{SF}}\left(r_{+}^{\mathrm{SF}}+b\right)}} \tan ^{-1} \frac{a}{\sqrt{r_{+}^{\mathrm{SF}}\left(r_{+}^{\mathrm{SF}}+b\right)}}\right] \\
\times\left[1-\frac{b r_{-}^{\mathrm{SF}}}{a \sqrt{r_{-}^{\mathrm{SF}}\left(r_{-}^{\mathrm{SF}}+b\right)}} \tan ^{-1} \frac{a}{\sqrt{r_{-}^{\mathrm{SF}}\left(r_{-}^{\mathrm{SF}}+b\right)}}\right]
\end{aligned}
$$

and

$$
\begin{aligned}
& \mathcal{S}_{+}{ }^{\mathrm{SF}}+\mathcal{S}_{-}{ }^{\mathrm{SF}}=2 \pi \mathcal{M} r_{+}{ }^{\mathrm{SF}} \\
& \times\left[1-\frac{b r_{+}^{\mathrm{SF}}}{a \sqrt{r_{+}^{\mathrm{SF}}\left(r_{+}^{\mathrm{SF}}+b\right)}} \tan ^{-1} \frac{a}{\sqrt{r_{+}^{\mathrm{SF}}\left(r_{+}^{\mathrm{SF}}+b\right)}}\right] \\
& +2 \pi \mathcal{M} r_{-} \mathrm{SF}\left[1-\frac{b r_{-}^{\mathrm{SF}}}{a \sqrt{r_{-}^{\mathrm{SF}}\left(r_{-}^{\mathrm{SF}}+b\right)}} \tan ^{-1} \frac{a}{\sqrt{r_{-}^{\mathrm{SF}}\left(r_{-}^{\mathrm{SF}}+b\right)}}\right]
\end{aligned}
$$

It also implies that the entropy product and entropy sum for $\mathcal{H}^{ \pm}$in SF of Sen BH are not universal.

For completeness, we also compute the irreducible mass of Sen $\mathrm{BH}$ for $\mathcal{H}^{ \pm}$in SF reads

$$
\begin{aligned}
& \mathcal{M}_{\mathrm{irr}, \pm}^{\mathrm{SF}} \\
& =\sqrt{\frac{\mathcal{M} r_{ \pm}^{\mathrm{SF}}}{2}\left[1-\frac{b r_{ \pm}^{\mathrm{SF}}}{a \sqrt{r_{ \pm}^{\mathrm{SF}}\left(r_{ \pm}^{\mathrm{SF}}+b\right)}} \tan ^{-1} \frac{a}{\sqrt{r_{ \pm}^{\mathrm{SF}}\left(r_{ \pm}^{\mathrm{SF}}+b\right)}}\right]} .
\end{aligned}
$$

For our record, we find the irreducible mass product in SF:

$$
\begin{aligned}
& \mathcal{M}_{\text {irr },+}{ }^{\mathrm{SF}} \mathcal{M}_{\mathrm{irr},-}{ }^{\mathrm{SF}}=\frac{\mathcal{J}}{2} \\
& \times \sqrt{\left[1-\frac{b r_{+}^{\mathrm{SF}}}{a \sqrt{r_{+}^{\mathrm{SF}}\left(r_{+}^{\mathrm{SF}}+b\right)}} \tan ^{-1} \frac{a}{\sqrt{r_{+}^{\mathrm{SF}}\left(r_{+}^{\mathrm{SF}}+b\right)}}\right]} \\
& \times \sqrt{\left[1-\frac{r_{-}^{\mathrm{SF}} b}{a \sqrt{r_{-}^{\mathrm{SF}}\left(r_{-}^{\mathrm{SF}}+b\right)}} \tan ^{-1} \frac{a}{\sqrt{r_{-}^{\mathrm{SF}}\left(r_{-}^{\mathrm{SF}}+b\right)}}\right]} .
\end{aligned}
$$

and the sum of irreducible mass is

$$
\begin{aligned}
& \mathcal{M}_{\text {irr },+}{ }^{\mathrm{SF}}+\mathcal{M}_{\text {irr, }-}{ }^{\mathrm{SF}}=\sqrt{\frac{\mathcal{M} r_{+}^{\mathrm{SF}}}{2}} \\
& \times \sqrt{\left[1-\frac{b r_{+}^{\mathrm{SF}}}{a \sqrt{r_{+}^{\mathrm{SF}}\left(r_{+}^{\mathrm{SF}}+b\right)}} \tan ^{-1} \frac{a}{\sqrt{r_{+}^{\mathrm{SF}}\left(r_{+}^{\mathrm{SF}}+b\right)}}\right]}
\end{aligned}
$$

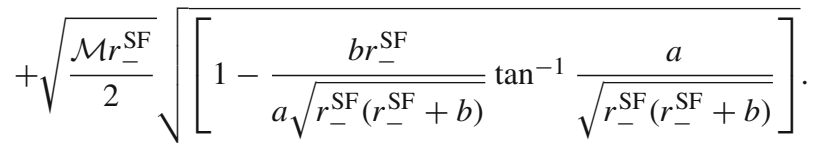

It seems that they both are not universal. It is obvious because irreducible mass depends on area. It is true that area product, entropy product and irreducible mass product gives same result because they are identical. For our record we computed them separately.

\section{Discussion}

In this work, we have examined various thermodynamic products for rotating charged black hole solution in four dimensional heterotic string theory. We have considered both EF and SF. In the EF, we have shown that the "area product" and "entropy product" are universal, while the "area sum" and the "entropy sum" are not! In the SF, we have shown that the "area product", "entropy product", "area sum" and "entropy sum" do not manifested any universal character because they all are depends on ADM mass parameter. We also showed that every $\mathrm{BH}$ thermodynamical variable, other than the mass $(M)$, the angular momentum $(J)$ and the charge $(Q)$ parameter, can form a quadratic equation whose roots are contained the three basic parameters $M, J, Q$. For completeness, we have derived the Smarr mass formula and Christodoulou's irreducible mass formula for Sen BH in the EF. Finally, we showed that the four laws of $\mathrm{BH}$ mechanics satisfied on both the horizons $\mathcal{H}^{ \pm}$.

Based on the thermodynamic relations, we also derived the area bound and entropy bound for all the horizons. Furthermore, we calculated the irreducible mass bound for this type of $\mathrm{BH}$. These formulas are expected to be useful to understanding the microscopic nature of BH entropy (both exterior and interior). Again, the entropy products of inner horizon and outer horizons could be used to determine whether the classical BH entropy could be written as a Cardy formula (see Appendix B), giving some evidence for a holographic description of BH/CFT correspondence [22]. The above thermodynamic properties including the Hawking temperature and area of both the horizons may therefore be expected to play a crucial role to understanding the $\mathrm{BH}$ entropy at the microscopic level. 
There has been compelling evidence by astrophysically that BH's have EH [30] and it is also true that the EH's are thermodynamically stable with respect to axi-symmetric perturbations $[31,32]$. Whereas there is no strong or weak evidence that BH's have $\mathrm{CH}$ by astrophysically but analytically has strong evidence that BH's possesses $\mathrm{CH}$ in addition with $\mathrm{EH}$, and it is also well known by fact that $\mathrm{CH}$ is thermodynamically unstable by axi-symmetric perturbations [33]. So still now it is unclear to us whether the $\mathrm{CH}$ thermodynamic results have a real astrophysical significance just as the event horizon does [30]. So, it will be a little bit help us to understanding the interior physics of Sen $\mathrm{BH}$ to clarify what the inner thermodynamics physically means. It may quite plausible that this interior physics could help us to understanding the interior BH entropy.

Acknowledgments I would like to thank the Editor for his patience and anonymous referee for his helpful suggestions.

Open Access This article is distributed under the terms of the Creative Commons Attribution 4.0 International License (http://creativecomm ons.org/licenses/by/4.0/), which permits unrestricted use, distribution, and reproduction in any medium, provided you give appropriate credit to the original author(s) and the source, provide a link to the Creative Commons license, and indicate if changes were made. Funded by SCOAP ${ }^{3}$.

\section{Appendix A}

\begin{tabular}{|c|c|c|c|}
\hline Parameter & $\mathrm{KN} \mathrm{BH}$ & Kerr BH & Sen BH \\
\hline$r_{ \pm}:$ & $M \pm \sqrt{M^{2}-a^{2}-Q^{2}}$ & $M \pm \sqrt{M^{2}-a^{2}}$ & $M-\frac{Q^{2}}{2 M} \pm \sqrt{\left(M-\frac{Q^{2}}{2 M}\right)^{2}-a^{2}}$ \\
\hline$\sum r_{i}:$ & $2 M$ & $2 M$ & $2 M-\frac{Q^{2}}{M}$ \\
\hline$\prod r_{i}:$ & $a^{2}+Q^{2}$ & $a^{2}$ & $a^{2}$ \\
\hline $\mathcal{A}_{ \pm}:$ & $4 \pi\left(r_{ \pm}^{2}+a^{2}\right)$ & $4 \pi\left(2 M^{2} \pm 2 \sqrt{M^{4}-J^{2}}\right)$ & $8 \pi M r_{ \pm}$ \\
\hline$\sum \mathcal{A}_{i}$ & $8 \pi\left(2 M^{2}-Q^{2}\right)$ & $16 \pi M^{2}$ & $8 \pi\left(2 M^{2}-Q^{2}\right)$ \\
\hline$\prod \mathcal{A}_{i}:$ & $(8 \pi)^{2}\left(J^{2}+\frac{Q^{4}}{4}\right)$ & $(8 \pi J)^{2}$ & $(8 \pi J)^{2}$ \\
\hline$S_{ \pm}:$ & $\pi\left(r_{ \pm}^{2}+a^{2}\right)$ & $\pi\left(r_{ \pm}^{2}+a^{2}\right)$ & $2 \pi M r_{ \pm}$ \\
\hline$\sum \mathcal{S}_{i}:$ & $2 \pi\left(2 M^{2}-Q^{2}\right)$ & $4 \pi M^{2}$ & $2 \pi\left(2 M^{2}-Q^{2}\right)$ \\
\hline$\prod \mathcal{S}_{i}$ & $(2 \pi)^{2}\left(J^{2}+\frac{Q^{4}}{4}\right)$ & $(2 \pi J)^{2}$ & $(2 \pi J)^{2}$ \\
\hline$\kappa_{ \pm}:$ & $\frac{r_{ \pm}-r_{\mp}}{2\left(r_{ \pm}^{2}+a^{2}\right)}$ & $\frac{r_{ \pm}-r_{\mp}}{2\left(r_{ \pm}^{2}+a^{2}\right)}$ & $\frac{r_{ \pm}-r_{\mp}}{4 M r_{ \pm}}$ \\
\hline$\sum \kappa_{i}:$ & $\frac{4 M\left(a^{2}+Q^{2}-M^{2}\right)}{\left(4 J^{2}+Q^{4}\right)}$ & $\frac{a^{2}-M^{2}}{a J}$ & $\frac{4 J^{2}-\left(2 M^{2}-Q^{2}\right)^{2}}{4 M J^{2}}$ \\
\hline$\prod \kappa_{i}:$ & $\frac{a^{2}+Q^{2}-M^{2}}{4 M^{2}\left(a^{2}+Q^{2}\right)}$ & $\frac{a^{2}-M^{2}}{4 J^{2}}$ & $\frac{4 J^{2}-\left(2 M^{2}-Q^{2}\right)^{2}}{(4 M J)^{2}}$ \\
\hline$T_{ \pm}:$ & $\frac{r_{ \pm}-r_{\mp}}{4 \pi\left(r_{ \pm}^{2}+a^{2}\right)}$ & $\frac{r_{ \pm}-r_{\mp}}{4 \pi\left(r_{ \pm}^{2}+a^{2}\right)}$ & $\pm \frac{\sqrt{\left(2 M^{2}-Q^{2}\right)^{2}-4 J^{2}}}{4 \pi M\left[\left(2 M^{2}-Q^{2}\right) \pm \sqrt{\left(2 M^{2}-Q^{2}\right)^{2}-4 J^{2}}\right]}$ \\
\hline $\begin{array}{l}\sum T_{i}: \\
\prod T_{i}:\end{array}$ & $\begin{array}{l}\frac{a^{2}+Q^{2}-M^{2}}{2 \pi M\left(a^{2}+Q^{2}\right)} \\
\frac{a^{2}+Q^{2}-M^{2}}{(4 \pi M)^{2}\left(a^{2}+Q^{2}\right)}\end{array}$ & $\begin{array}{l}\frac{a^{2}-M^{2}}{2 \pi a J} \\
\frac{a^{2}-M^{2}}{(4 \pi J)^{2}}\end{array}$ & $\begin{array}{l}4 \pi M\left[\left(2 M^{2}-Q^{2}\right) \pm \sqrt{\left.\left(2 M^{2}-Q^{2}\right)^{2}-4 J^{2}\right]}\right. \\
\frac{4 J^{2}-\left(2 M^{2}-Q^{2}\right)^{2}}{8 \pi M J^{2}} \\
\frac{4 J^{2}-\left(2 M^{2}-Q^{2}\right)^{2}}{(8 \pi J M)^{2}}\end{array}$ \\
\hline$M_{\mathrm{irr}, \pm}:$ & $\sqrt{\frac{\mathcal{A}_{ \pm}}{16 \pi}}$ & $\sqrt{\frac{\mathcal{A}_{ \pm}}{16 \pi}}$ & $\sqrt{\frac{\mathcal{A}_{ \pm}}{16 \pi}}$ \\
\hline$\sum M_{\mathrm{irr}, \pm}^{2}:$ & $M^{2}$ & $M^{2}$ & $M^{2}-\frac{Q^{2}}{2}$ \\
\hline$\prod M_{\mathrm{irr}, \pm}:$ & $\sqrt{\frac{J^{2}+\frac{Q^{4}}{4}}{4}}$ & $\frac{J}{2}$ & $\frac{J}{2}$ \\
\hline$\Omega_{ \pm}:$ & $\frac{a}{2 M r_{ \pm}-Q^{2}}$ & $\frac{a}{2 M r_{ \pm}}$ & $\frac{a}{2 M r_{ \pm}}$ \\
\hline$\sum \Omega_{i}$ & $\frac{2 a\left(2 M^{2}-Q^{2}\right)}{4 J^{2}+Q^{4}}$ & $\frac{1}{a}$ & $\frac{2 M^{2}-Q^{2}}{2 a M^{2}}$ \\
\hline$\prod \Omega_{i}:$ & $\frac{a^{2}}{4 J^{2}+Q^{4}}$ & $\frac{1}{4 M^{2}}$ & $\frac{1}{4 M^{2}}$ \\
\hline$E_{ \pm}:$ & $\pm \sqrt{M^{2}-a^{2}-Q^{2}}$ & $\pm \sqrt{M^{2}-a^{2}}$ & $\pm \sqrt{\left(2 M^{2}-Q^{2}\right)^{2}-4 J^{2}}$ \\
\hline$\sum E_{i}:$ & 0 & 0 & 0 \\
\hline$\prod E_{i}$ & $-\left(M^{2}-a^{2}-Q^{2}\right)$ & $-\left(M^{2}-a^{2}\right)$ & $-\left[\left(2 M^{2}-Q^{2}\right)^{2}-4 J^{2}\right]$ \\
\hline$r_{+}=r_{-}:$ & $M^{2}=a^{2}+Q^{2}$ & $M^{2}=a^{2}$ & $a=M-\frac{Q^{2}}{2 M}$ \\
\hline
\end{tabular}




\section{Appendix B}

Here we shall derive the central charges $c_{L}$ and $c_{R}$ of the left and right moving sectors of the dual CFT in Sen/CFT correspondence. We shall prove that the central charges of the left and right moving sectors are same i.e. $c_{L}=c_{R}$ for Sen $\mathrm{BH}$. Also we shall derive the dimensionless temperature of microscopic CFT from the above thermodynamic relations. Furthermore using Cardy formula, we shall derive the left and right moving entropies in 2D CFT.

In terms of $r_{+}$and $r_{-}$, we can write the ADM mass and spin parameter as

$$
\begin{aligned}
& M=\frac{1}{4}\left[\left(r_{+}+r_{-}\right)+\sqrt{\left(r_{+}+r_{-}\right)^{2}+8 Q^{2}}\right] \\
& \text { and } a=\sqrt{r_{+} r_{-}} .
\end{aligned}
$$

Now the angular momentum can be written as

$$
J=\frac{\sqrt{r_{+} r_{-}}}{4}\left[\left(r_{+}+r_{-}\right)+\sqrt{\left(r_{+}+r_{-}\right)^{2}+8 Q^{2}}\right] .
$$

Moreover using $r_{+}$and $r_{-}$, we can write the entropy, Hawking temperature, angular velocity and electric potential for $\mathcal{H}^{+}$:

$$
\begin{aligned}
& S_{+}=\frac{\pi r_{+}\left[\left(r_{+}+r_{-}\right)+\sqrt{\left(r_{+}+r_{-}\right)^{2}+8 Q^{2}}\right] .}{T_{+}}=\frac{r_{+}-r_{-}}{2 \pi r_{+}\left[\left(r_{+}+r_{-}\right)+\sqrt{\left(r_{+}+r_{-}\right)^{2}+8 Q^{2}}\right]} . \\
& \Omega_{+}=\frac{2 \sqrt{r_{+} r_{-}}}{r_{+}\left[\left(r_{+}+r_{-}\right)+\sqrt{\left(r_{+}+r_{-}\right)^{2}+8 Q^{2}}\right]} . \\
& \phi_{+}=\frac{4 Q}{\left[\left(r_{+}+r_{-}\right)+\sqrt{\left(r_{+}+r_{-}\right)^{2}+8 Q^{2}}\right]} .
\end{aligned}
$$

Finally, using the symmetry of $r_{ \pm}$, one can obtain the following relations for the thermodynamic quantities at $\mathcal{H}^{-}$:

$$
\begin{gathered}
T_{-}=-\left.T_{+}\right|_{r_{+} \leftrightarrow r_{-}}, S_{-}=\left.S_{+}\right|_{r_{+} \leftrightarrow r_{-}}, \\
\Omega_{-}=\left.\Omega_{+}\right|_{r_{+} \leftrightarrow r_{-}}, \Phi_{-}=\left.\Phi_{+}\right|_{r_{+} \leftrightarrow r_{-}} .
\end{gathered}
$$

The first law of BH thermodynamics can be rewritten as in terms of left and right moving modes of dual CFT:

$$
\begin{aligned}
\frac{\mathrm{d} M}{2} & =T_{L} \mathrm{~d} S_{L}+\Omega_{L} \mathrm{~d} J+\Phi_{L} \mathrm{~d} Q . \\
& =T_{R} \mathrm{~d} S_{R}+\Omega_{R} \mathrm{~d} J+\Phi_{R} \mathrm{~d} Q .
\end{aligned}
$$

with the definitions $\beta_{R, L}=\beta_{+} \pm \beta_{-}, \beta_{ \pm}=\frac{1}{T_{+}}, \Omega_{R, L}=$ $\frac{\beta_{+} \Omega_{+} \pm \beta_{-} \Omega_{-}}{2 \beta R, L}, \Phi_{R, L}=\frac{\beta_{+} \Phi_{+} \pm \beta_{-} \Phi_{-}}{2 \beta R, L}$ and $S_{R, L}=\frac{\left(S_{+} \mp S_{-}\right)}{2}$.
Using the above relations, we find

$$
\begin{aligned}
& T_{L}=\frac{1}{2 \pi\left[\left(r_{+}+r_{-}\right)+\sqrt{\left(r_{+}+r_{-}\right)^{2}+8 Q^{2}}\right]}, \\
& T_{R}=\frac{r_{+}-r_{-}}{2 \pi\left(r_{+}+r_{-}\right)\left[\left(r_{+}+r_{-}\right)+\sqrt{\left(r_{+}+r_{-}\right)^{2}+8 Q^{2}}\right]} \\
& S_{L}=\frac{\pi\left(r_{+}+r_{-}\right)}{4}\left[\left(r_{+}+r_{-}\right)+\sqrt{\left(r_{+}+r_{-}\right)^{2}+8 Q^{2}}\right] \\
& S_{R}=\frac{\pi\left(r_{+}-r_{-}\right)}{4}\left[\left(r_{+}+r_{-}\right)+\sqrt{\left(r_{+}+r_{-}\right)^{2}+8 Q^{2}}\right] \\
& \Omega_{L}=0, \\
& \Omega_{R}=\frac{2 \sqrt{r_{+} r_{-}}}{\left(r_{+}+r_{-}\right)\left[\left(r_{+}+r_{-}\right)+\sqrt{\left(r_{+}+r_{-}\right)^{2}+8 Q^{2}}\right]} \\
& \Phi_{L}=\frac{2 Q}{\left[\left(r_{+}+r_{-}\right)+\sqrt{\left(r_{+}+r_{-}\right)^{2}+8 Q^{2}}\right]}, \\
& \Phi_{R}=\frac{2 Q}{\left[\left(r_{-}+r_{+}\right)+\sqrt{\left(r_{-}+r_{+}\right)^{2}+8 Q^{2}}\right]} .
\end{aligned}
$$

Using Eqs. $(127,128)$ and setting $\mathrm{d} Q=0$, we obtain the first law of left and right sectors:

$\mathrm{d} J=\frac{T_{L}}{\Omega_{R}-\Omega_{L}} \mathrm{~d} S_{L}-\frac{T_{R}}{\Omega_{R}-\Omega_{L}} \mathrm{~d} S_{R}$.

This gives the dimensionless temperature of the left and right moving sectors of the dual CFT correspondence and are given by

$T_{L, R}^{J}=\frac{T_{L, R}}{\Omega_{R}-\Omega_{L}}$.

which is exactly the microscopic temperature of the CFT and found to be for Sen BH

$T_{L, R}^{J}=\frac{r_{+} \pm r_{-}}{4 \pi \sqrt{r_{+} r_{-}}}$.

Now we find the central charges [22] in left and right moving sectors of the Sen/CFT correspondence via the Cardy formula reads

$S_{L, R}^{J}=\frac{\pi^{2}}{3} c_{L, R}^{J} T_{L, R}^{J}$.

Therefore the central charges of dual CFT should be

$c_{L}^{J}=c_{R}^{J}=12 J$.

which is exactly same as Kerr BH [34] and $\mathrm{KN} \mathrm{BH}$ [22]. This observation tells us that Sen BH is dual to a $c_{L}=c_{R}=12 \mathrm{~J}$ 2D CFT at temperature $\left(T_{L}, T_{R}\right)$ for each value of $M$ and $J$. 
In the extremal limit $r_{+}=r_{-}$, the above expressions reduce to

$$
\begin{aligned}
& T_{L}=\frac{1}{4 \pi\left[r_{+}+\sqrt{r_{+}^{2}+2 Q^{2}}\right]}, \quad T_{R}=0 \\
& S_{L}=\pi r_{+}\left[r_{+}+\sqrt{r_{+}^{2}+2 Q^{2}}\right], \quad S_{R}=0 \\
& \Omega_{L}=0, \quad \Omega_{R}=\frac{1}{2\left[r_{+}+\sqrt{r_{+}^{2}+2 Q^{2}}\right]} \\
& \Phi_{L}=\Phi_{R}=\frac{Q}{\left[r_{+}+\sqrt{r_{+}^{2}+2 Q^{2}}\right]} . \\
& T_{L}^{J}=\frac{1}{2 \pi}, \quad T_{R}^{J}=0 .
\end{aligned}
$$

this left moving temperature is actually Frolov-Thorn temperature, and finally the central charge for extremal Sen $\mathrm{BH}$ :

$c_{L}^{J}=12 J$.

Therefore, we obtain the microscopic entropy via the Cardy formula in chiral dual CFT:

$S_{\text {micro }}=\frac{\pi^{2}}{3} c_{L}^{J} T_{L}^{J}=2 \pi J$.

which is perfectly agreement with macroscopic BekensteinHawking entropy of the extreme Sen BH.

\section{References}

1. J.D. Bekenstein, Lett. Nuov. Cimento 4, 737 (1972)

2. J.D. Bekenstein, Phys. Rev. D 7, 2333 (1973)

3. J.D. Bekenstein, Phys. Rev. D 9, 3292 (1974)

4. J.M. Bardeen, B. Carter, S.W. Hawking, Commun. Math. Phys. 31, $161(1973)$
5. P.A. Blaga, C. Blaga, Class. Quant. Grav. 18, 3893-3905 (2001)

6. R. Casadio, B. Harms, Mod. Phys. Lett. A 14, 1098 (1999)

7. L. Smarr, Phys. Rev. Lett. 30, 71 (1973) [Erratum-ibid. 30, 521 (1973)]

8. M. Ansorg, J. Hennig, Phys. Rev. Lett. 102, 221102 (2009)

9. M. Visser, J. High Energy Phys. 06, 023 (2012)

10. P. Pradhan, Eur. Phys. J. C 74, 2887 (2014)

11. P. Pradhan, Phys. Lett. B 747, 64 (2015)

12. A. Curir, Nuovo Cimento 51B, 262 (1979)

13. M. Cvetič, D. Youm, Phys. Rev. D 54, 2612 (1996)

14. M. Cvetič, F. Larsen, Nucl. Rhys. B 506, 107 (1997)

15. M. Cvetič, F. Larsen, Phys. Rev. D 56, 4994 (1997)

16. M. Cvetič, G.W. Gibbons, C.N. Pope, Phys. Rev. Lett. 106, 121301 (2011)

17. M. Cvetič, H. Lü, C.N. Pope, Phys. Rev. D 88, 044046 (2013)

18. A. Castro, M.J. Rodriguez, Phys. Rev. D 86, 024008 (2012)

19. A.M. Ghezelbash, H.M. Siahaan, Class. Quant. Grav. 30, 135005 (2013)

20. S. Detournay, Phys. Rev. Lett. 109, 031101 (2012)

21. V. Faraoni, A.F.Z. Moreno, Phys. Rev. D. 88, 044011 (2013)

22. B. Chen, S.X. Liu, J.J. Zhang, J. High Energy Phys. 017, 1211 (2012)

23. S. Chandrashekar, The Mathematical Theory of Black Holes (Clarendon Press, Oxford, 1983)

24. A. Sen, Phys. Rev. Lett. 69, 1006 (1992)

25. A. Sen, Nucl. Phys. B 440, 421 (1995)

26. A. Komar, Phys. Rev. 113, 934 (1959)

27. R. Penrose, Ann. N. Y. Acad. Sci. 224, 125 (1973)

28. W. Xu, J. Wang, X. Meng, Phys. Lett. B 746, 53 (2015)

29. D. Christodoulou, R. Ruffini, Phys. Rev. D. 4, 3552 (1971)

30. R. Narayan, Astron. Geophys. 44, 6.22 (2003)

31. O. Kaburki, I. Okamoto, Phys. Rev. D. 43, 340 (1991)

32. I. Okamoto, O. Kaburki, Mon. Not. R. Astron. Soc. 255, 539 (1992)

33. V.P. Frolov, I.D. Novikov, Black Hole Physics (Springer, Netherlands, 1989)

34. T. Hartman, K. Murata, T. Nishioka, A. Strominger, J. High Energy Phys. 04, 019 (2009) 